\title{
Justice denied: Gender and Motherhood Pay Gaps in Poland
}

\author{
Kinga Wysieńska-Di Carlo and Zbigniew Karpiński \\ Institute of Philosophy and Sociology \\ Polish Academy of Sciences*
}

October 2019

\begin{abstract}
Motherhood wage penalties and the reasons for their persistence have received a great deal of attention in the sociological literature over the past twenty years. Rarely do these studies present results from Central and Eastern Europe, however. Using data from the nationally representative Polish Panel Survey (POLPAN) for the years 1988-2018, we estimate the size of the motherhood penalty in Poland. We show that while mothers and women in Poland incur significant wage penalties compared to men, even after controlling for a range of productivity and human capital characteristics, mothers do not earn less than women with no children. In order to address the argument that such disparities endure because they are viewed as just, we contrast our results with those of a multifactorial (vignette) study of fair earnings. The latter shows that such wage penalties are not perceived as just. In fact, we find no evidence of perceived just penalties for women and mothers with the same characteristics as men and fathers. Our results provide insights into the ongoing discussion of principles of legitimacy of inequality and perceptions of fairness.
\end{abstract}

\section{Funding}

The work was supported by the Polish National Science Center (grant numbers 2015/19/B/HS6/03169 and 2017/25/B/HS6/02697).

\section{Acknowledgements}

Earlier versions of this paper were presented at 2018 Annual American Sociological Association Meeting in Philadelphia and 2019 Motherhood Penalty Seminar in Warsaw. We thank the participants of these events for their valuable feedback. We also thank Anna Kiersztyn for her help with income adjustments.

${ }^{*}$ Corresponding author: kwysienska@ifispan.waw.pl. This manuscript is a work in progress. Please do not quote without permission. 


\section{Introduction}

Both comparative (e.g., Osberg and Smeeding 2006; Kelley and Zagórski 2004) and national (Kołczyńska and Merry 2016) studies have explored links between people's assessments of fair earnings for a given occupation and their perceptions of how much incumbents of those occupations actually earn. Most of these studies use responses to a set of items listing a number of generic occupations (e.g., doctor in general practice, skilled factory worker, lawyer). Respondents are tasked with assessing how much they think persons in these occupations do earn and how much they should earn. The relationships between these two subjective assessments are then used to infer norms of propriety (Walker, Thomas, and Zelditch 1986) - that is, according to individuals or groups, what income differences are "fair" or "deserved?"

This method of measuring norms underlying the legitimacy of inequality has its advantages. For example, it allows for reducing a host of errors caused by burdening respondents with estimating the size of income disparities (Osberg and Smeeding 2006; Eriksson and Simpson 2012). It is problematic, however, when one wants to establish whether, and to what extent, gender norms and parenthood status play a role in people's assessments of fair inequalities. This is because, first, gender and/or parenthood status of a person in a given occupation is not specified in these survey items. Second, the use of such general occupational descriptions doesn't reflect the gendered nature of workplaces. Third, this approach cannot assess the effects of productivity and human capital characteristics, which are often associated with gender wage disparities and motherhood penalties. Finally, asking respondents directly about how much a person in a given occupation earns, and how much such a person should earn, is prone to bias due to the activation of certain cognitive heuristics and uncertainty regarding the situation (Markovsky 1988).

We therefore use an alternative, two-stage approach. First, using a unique data set, the Polish Panel Survey, or POLPAN (Słomczyński et al. 2016), we assess gender and motherhood penalties, controlling for occupational status and productivity and human capital characteristics (e.g., seniority, cumulative job experience, education).

These results, which show that Polish women and mothers do indeed incur significant wage penalties, motivate and provide context for interpreting the results of this study's second phase: a multifactorial (vignette) experiment (Auspurg and Hinz 2015) conducted on a representative sample of the Polish working population at the end of 2017. The vignette study was designed to measure the impact of gender and parenthood status on justice evaluations of earnings in a manner that allows for comparisons with our POLPAN results. Specifically, in constructing the population of vignettes, we used productivity and human capital characteristics corresponding to those measured in POLPAN. We also selected occupations based on occupational prestige categories using the same prestige scale as those used in POLPAN.

Our paper makes three contributions. First, it presents robust estimates of motherhood penalties and fatherhood premia in Poland. Previous studies either 
relied on data that rendered it difficult to gauge the effects of parenthood in Poland (Cukrowska-Torzewska and Lovasz 2016) or used limited cross-sectional data (that included Poland) to test hypotheses on general relationships between social policies and/or attitudes and motherhood penalties/"the family wage gaps" (Budig, Misra, and Boeckmann 2012, 2016; Cukrowska-Torzewska and Lovasz 2016). Apart from these studies, perhaps due in part to assumptions about "countries in transition" and "women in transition" being a specific case (Cukrowska-Torzewska 2016; Mikucka 2013), Poland was left out of crossnational comparisons of motherhood penalties' correlates. We show motherhood penalties and fatherhood premia patterns in Poland and compare them to those in other countries.

Second, we provide robust data regarding perceptions of just earnings for men and women of varying parenthood status and job and human capital characteristics. Although studies of the effects of gender on fair earnings (Auspurg, Hinz, and Sauer 2017; Jasso and Webster 1997, 1999) have included human capital characteristics, they have not analyzed the effects of those characteristics in the context of parenthood status in general and/or the number of children in particular. Our analysis shows that when job experience is held constant, neither parenthood status nor the number of children affects estimates of just earnings.

Third, while we do not test any specific hypotheses — as our study is exploratory - we do provide evidence questioning the notion that what people perceive as just is a direct function of the actual situation is (Jost, Banaji, and Nosek 2004; Kelley and Zagórski 2004). Our evidence suggests that the "justification principle" needs to be appended with additional scope conditions, especially when observed or experienced inequalities are not simply associated with meritocratic variables (e.g., occupational prestige, education, or effort). Linking inequalities to such ascribed categories as gender or race, or other non-meritocratic characteristics, such as parenthood status, may weaken the conviction of the propriety of a given distribution in contemporary societies.

The structure of the remainder of this paper is as follows. We first discuss arguments regarding the sources of legitimacy of earning gaps in general, and gender and motherhood penalties in particular. We then turn to the Polish context and present our estimates of the actual and just motherhood penalties and gender wage gaps in Poland. Finally, we interpret our results in the context of existing theories and research.

\section{Legitimacy of earning gaps}

Studies show that despite growing income inequalities in many western and post-communist countries, support for redistribution has not increased (Kelly and Enns 2010; Kenworthy and McCall 2008). This observation contradicts the "median-voter" hypothesis, which posits that self-interest leads to higher support for redistribution when inequalities are on the rise. The search for explanations of such results has led to a number of hypotheses regarding confounding and 
intervening factors. One such hypothesis claims that actual income inequalities, when observed, are viewed as legitimized.

This hypothesis is rooted in the notion that people need to make sense of disparities around them and therefore perceive the status quo as "good, fair, natural, desirable, and even inevitable" (Jost, Banaji, and Nosek 2004, p. 887). This need is so strong that both high and low socioeconomic status group members share the rationalizations they use, regardless of whether they favor them or not. Moreover, likely events are judged as more desirable than less likely or unlikely ones. Expected inequalities (i.e., those linked to characteristics prone to produce differential outcomes) are more likely to be justified than those that are less commonly experienced and are not anticipated to have impact on observable outcomes.

Consequently, to achieve or restore a sense of a just world, people interpret inequities as deserved based on attributes that are often correlated with them, such as education and effort (Frank 2016; Heiserman and Simpson 2017) or gender and race (Ridgeway et al. 1998). It has been theorized that it is either through the process of doubly dissimilar encounters (Ridgeway et al. 1998) or through self-evaluations (Della Fave 1986) that actual disparities turn into "proper" (i.e., legitimized by an individual) ones (Walker, Thomas, and Zelditch 1986).

Research on attitudes towards income inequalities seems to support this hypothesis. Across countries, respondents agree on which occupations should pay the most, and the perceptions of which occupations do pay the most is the strongest predictor of these rankings (Kelley and Zagórski 2004; Osberg and Smeeding 2006). Moreover, the rise in acceptance of occupational income inequalities seems to be most prevalent in Central-European post-communist countries, where the actual and perceived income differences grew rapidly during their transitions (Kelley and Zagórski 2004; Kołczyńska and Merry 2016).

\subsection{Motherhood penalties and their legitimacy}

Women earn less than men even after controlling for a host of (indirect) productivity measures (see Charles 2011). In addition, mothers in the US (e.g., England et al. 2016; Staff and Mortimer 2012), and Australia (e.g., Cooke 2014) suffer additional wage and occupational prestige losses (Abendroth, Huffman, and Treas 2014). The size of motherhood penalties, understood here as wage losses incurred by mothers compared to women with no children, and fatherhood premia (defined, analogously, as wage increases of fathers compared to men with no children) vary across countries (e.g., Cooke 2014), welfare regimes (e.g., Aisenbrey and Fasang 2017; Budig, Misra, and Boeckmann 2016), and by income and occupational prestige (England et al. 2016; Cooke 2014). In general, however, men accrue modest fatherhood bonuses, whereas motherhood predicts a significant earnings penalty for women.

Researchers have proposed that these gaps and penalties are legitimized due to cross-culturally held beliefs regarding gender and motherhood as status characteristics (Ridgeway and Correll 2004). That is, being a woman, like having 
non-white skin color or being less educated, is linked to a set of universally shared expectations regarding competences, motivations, and general worth, while being a mother is perceived as especially incongruent with being an "ideal worker." Numerous studies show that mothers indeed are viewed as less workcommitted and competent workers than are childless women or men with or without children (Correll, Benard, and Paik 2007; Cuddy, Fiske, and Glick 2004; Fuegen et al. 2004).

Gender and motherhood as status characteristics (akin to race or ethnicity) lead to divergent performance expectations and evaluations, double standards of competence assessments, and unequal reward expectations. The legitimacy of gender and motherhood effects is thus a self-fulfilling prophecy. Women, particularly mothers, are expected to be "lower quality" employees compared to men and fathers. They therefore have less opportunity to perform and their performance is usually rated as less satisfying than that of men. As reward expectations are linked to status characteristics and performance, women are expected to be and are rewarded less than men. The more often such inequality is experienced, the stronger the belief in its propriety and validity. Motherhood and gender as status characteristics hence jointly affect perceptions of job-related competence and suitability, which can partly explain the existence and persistence of motherhood pay penalties.

\subsection{Gender and Motherhood Penalties in Poland}

There is limited evidence on parents' and non-parents' outcomes on the labor market in Poland. Cukrowska-Torzewska (2016), using longitudinal data on women 18-40 years old between 2003 and 2009, finds evidence of a motherhood pay penalty, especially among mothers with multiple children. The magnitude of the penalty varies substantially depending on the model specifications, however, and the estimates are not always statistically significant. On the other hand, Cukrowska-Torzewska and Lovasz (2016), using data from the Polish Household Budget Surveys, conclude that fatherhood premia, rather than motherhood penalties, account for most of the observed gender pay gap. Budig, Misra, and Boeckmann (2012), using data from various sources, including the 2004 wave of the Polish Household Survey, find a motherhood penalty of $5.5 \%$ in Poland. The cross-sectional data employed in the latter three studies, and the short time-span in the former study, allow for correlating current parenthood status with current labor-market outcomes, but do not allow for a dynamic analysis of whether changes in these outcomes are associated with changes in the parenthood status over the course of the occupational career.

\section{Data}

To estimate gender and motherhood wage gaps in Poland throughout the occupational career, we use data from the Polish Panel Survey (POLPAN). POLPAN is a nationally representative longitudinal survey, started in 1988, with primary 
Table 1: Descriptive statistics for the POLPAN variables used in the analysis

\begin{tabular}{lrr}
\hline Variable & Mean & S.D. \\
\hline Female & 0.44 & 0.5 \\
& & \\
Degree of education (ref.: & Elementary) & \\
Tertiary & 0.25 & 0.4 \\
Post-secondary non-tertiary & 0.07 & 0.2 \\
Secondary & 0.31 & 0.5 \\
$\quad$ Vocational & 0.29 & 0.5 \\
Region (ref.: Central) & & \\
$\quad$ East & 0.19 & 0.4 \\
North-West & 0.15 & 0.4 \\
$\quad$ North & 0.15 & 0.4 \\
$\quad$ South & 0.22 & 0.4 \\
$\quad$ South-West & 0.09 & 0.3 \\
Year of birth & 1960.19 & 13.5 \\
Age & 40.55 & 10.7 \\
Rural & 0.30 & 0.5 \\
City & 0.33 & 0.5 \\
Married & 0.79 & 0.4 \\
Prestige & 38.62 & 21.5 \\
Hours & 44.43 & 9.3 \\
Tenure & 10.47 & 9.2 \\
Job experience & 17.85 & 10.6 \\
Children & 0.88 & 1.0 \\
Earnings & $1,863.62$ & $2,190.1$ \\
Adjusted earnings & $1,973.63$ & $1,624.4$ \\
\hline & & \\
\hline & &
\end{tabular}

focus on occupational structure and occupational mobility. It also covers a number of other topics, including political, social, and economic attitudes, friendship and other social relations (Słomczyński et al. 2016).

The POLPAN dataset includes 7,789 unique respondents and 18,656 unique respondent-wave combinations. Of these, 6,198 respondents (11,437 respondentwave combinations) were working at the time of their interviews. This subsample was further reduced to include only those respondents who participated in at least two waves of POLPAN. We also apply the following reductions in the size of our analysis sample: $(a)$ we focus only on individuals working full time to facilitate comparisons with the vignette study results (i.e., at least 30 hours per week and with a current job tenure of more than 3 months); and (b) we drop all cases with missing values on any of the variables used in the analysis. The final dataset thus includes 1,422 unique respondents and 4,159 respondent-wave combinations. Table 1 shows descriptive statistics for the variables used in the analysis. 
Figure 1: Sample structure in the POLPAN data

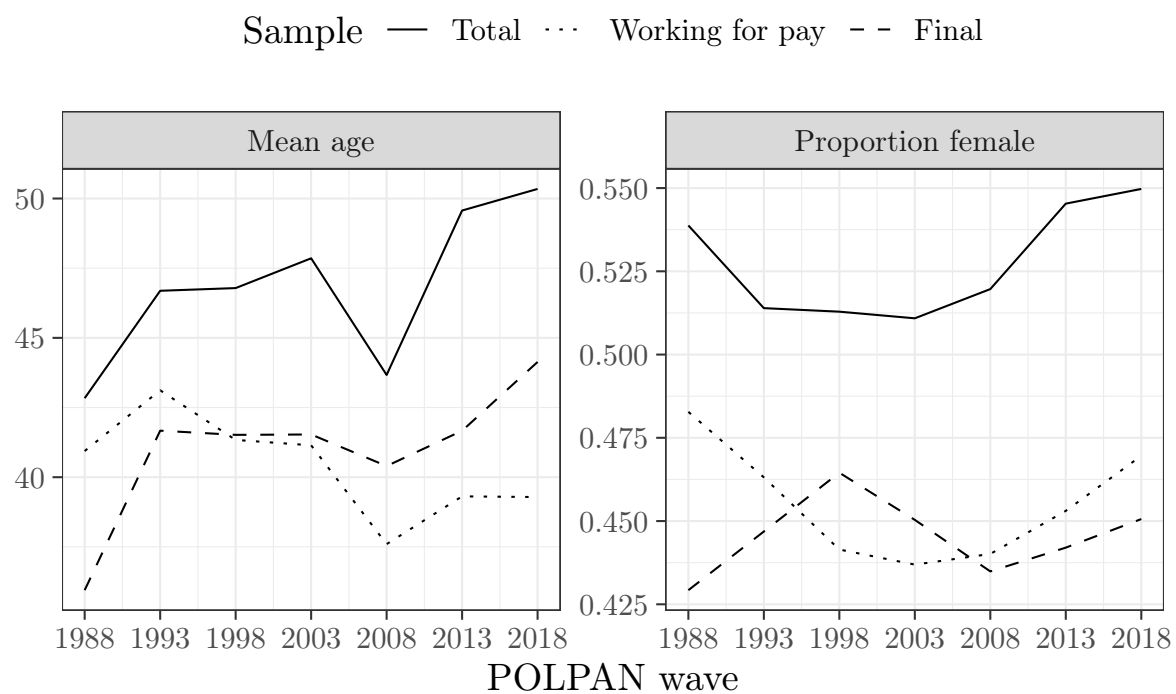

In addition, Figure 1 compares the age and gender structure of three samples: (a) the total POLPAN sample (represented by the solid line); (b) the sub-sample composed of all respondents who were working for pay (the dotted line); and (c) all individuals who were included in the final analysis (the dashed line). Respondents in the total sample, on average, are older and more likely to be male than are respondents in the two sub-samples.

Regarding the age composition, this is due to the fact that the total sample includes retired respondents who no longer work and therefore are not included in the two sub-samples. Also, the average age in the "final" sub-sample is increasing over time, whereas the average age in the "working for pay" sub-sample is decreasing slightly. This difference can be attributed to our decision to include in the final sample only those respondents who participated in at least two waves of POLPAN (so, by default, the youngest cohort is not included, see https: //polpan.org/en/data-and-documentation/methodology/); as well as our including only those who are employed full time (younger respondents are less likely to have full-time employment, see Kiersztyn 2016).

Regarding the gender composition, the overall difference between the subsamples and the general sample is attributable to women being less active in the labor force (Eurostat 2019) and the fact that mothers face a greater risk of unemployment, which itself is an indicator of inequality (Karpiński and Wysieńska-Di Carlo 2017; Wysieńska-Di Carlo and Karpiński 2015). 


\subsection{Dependent variable}

The natural logarithm of the self-reported net monthly on-the-job earnings, adjusted to 2018 amounts, is the dependent variable in our analysis. Comparisons of net monthly earnings distributions as reported by respondents match closely the estimates of on-the-job earnings reported in the Structure of Earnings Surveys conducted by the Polish Statistical Office (Kiersztyn 2014). In Poland, any tax deductions related to family or personal situation are applied at the end of the year, so monthly earnings as such do not depend on family size or situation. Gender pay gaps are defined as the ratio of the average earnings of women to the average earnings of men with the same characteristics. Below, the gaps are estimated using the regression coefficient for gender. Motherhood gender pay gaps are defined similarly - i.e., as the ratio of average earnings of mothers to those of similar fathers (using the regression coefficients for gender and gender's interaction with parental status). The motherhood wage penalty is estimated using the coefficient for the number of children and its interaction with gender. The fatherhood premium is estimated using the regression coefficient for the number of children.

\subsection{Independent variables}

Number of children, interacted with gender, is the key independent variable in our analysis. Apart from the 2018 wave, POLPAN participants were not asked explicitly about the number of children they had. Information about the number of children in preceding waves is based on data about household composition, using which we calculated the number of children up to 18 years of age who lived with the respondent during a given POLPAN wave.

In each wave of POLPAN, participants are asked detailed questions concerning their occupational and work history. They are asked about the job (or jobs) they held at the time of the interview, as well as all jobs they had during the five years between or prior to the interviews. For each reported job, participants are asked when (i.e., what year and month) the employment started, when it ended (if applicable), how many working hours a week it involved, etc. The jobs are then classified using the Polish Sociological Classification of Occupations (PSCO) and its accompanying scales of prestige, complexity, skill requirements, and material remuneration (see Domański, Słomczyński, and Sawiński 2009, for a detailed presentation of the classification and the scales). Hence, for each job reported by the respondents, we computed: (a) its duration in months; (b) the number of weekly working hours; and (c) scores on the occupational scales. Finally, using the information about job duration, we calculated overall job experience for each respondent.

We include a number of other characteristics in our model: gender; year of birth; age at the time of a given POLPAN wave; educational attainment; region; type of place of residence (rural area, small towns, large cities); and marital status. We define educational attainment as the highest degree of education obtained by the respondent; coded in this manner, it is a time-invariant 
characteristic.

Region is also a time-invariant characteristic in our analysis. Out of the 1,422 respondents included in our final sample, only 7 moved across regions between successive POLPAN waves. For the 7 respondents in our sample who did move, we assigned the region in which they stayed the longest.

As regards the type of place of residence, it is represented by two dummy variables: the first, "rural," is coded 1 for residents in rural areas and 0 otherwise; the second, "large city," is coded 1 for residents in cities with population at least 100,000 and 0 otherwise; residents in the smaller towns are the reference category.

Respondents' marital status is also represented as a dummy variable - it is coded 1 for respondents who are married or in a long-term relationship other than marriage, and 0 otherwise. Place of residence and marital status are timevarying variables.

\subsection{Analytical strategy}

Much of the research on motherhood penalties that uses longitudinal data has relied on fixed-effects methodology to estimate the size of these penalties. In these studies, the penalties are interpreted dynamically - that is, in terms of a change in a woman's earnings that can be attributed to a change in her motherhood status (i.e., the number of children). As we are interested in how parenthood affects one's earned income, as well as in gender pay gaps, our dataset is comprised of both female and male respondents. Gender pay gaps are an example of a between-person effect, which renders the fixed-effects approach of earlier research unsuitable for our purposes. We therefore apply a two-level hierarchical model, where multiple observations over time can be seen as nested within individuals.

At the first level, we model the log of net monthly earnings as a function of time-varying job characteristics, such as job experience, job prestige, weekly working hours, as well as of family circumstances, such as getting married or becoming a parent. At the second level, we model parameters of the lower-level earnings function as dependent upon individual characteristics - specifically, gender. This way, we are able not only to estimate how much, if at all, the impact of parenthood on earnings varies across gender categories, but also the average difference in logged earnings between men and women, net of all the productivity- and family-related characteristics included in the analysis.

Our initial model is "empty" in the sense that it contains only the intercept and error term. Note, however, that we allow the intercept to vary across individuals:

$$
\begin{aligned}
y_{i t} & =\beta_{0 i}+\epsilon_{i t} \\
\beta_{0 i} & =\gamma_{00}+u_{0 i}
\end{aligned}
$$

In the model, $y_{i t}$ represents the logged net monthly earnings and $\beta_{0 i}$ is the respondent-specific intercept. The intercept is expressed as a function of the 
overall intercept, $\gamma_{00}$ and an "error" term, representing how much respondent $i$ 's intercept departs from the overall one. The overall intercept is therefore the grand mean, taken over all respondents at all time points, and $u_{0 i}$ as the difference between the grand mean and the mean for respondent $i$. Variance of $u_{0 i}$ represents the part of the total variability in the dependent variable that can be attributed to differences between respondents; the remaining part, then, is due to differences, or changes, within respondents.

Respondent-specific intercept is a function of respondent-level characteristics, i.e., gender, year of birth, educational attainment, and region. Following Staff and Mortimer (2012), we also add to the model individual means of the time-varying variables:

$$
\begin{aligned}
& y_{i t}=\beta_{0 i}+\epsilon_{i t} \\
& \beta_{0 i}=\gamma_{00}+\gamma_{01} g_{i}+\gamma_{02} b_{i}+\gamma_{03} d_{i}+\gamma_{04} r_{i}+\sum_{k=5}^{K} \gamma_{0 k} w_{k, i}+u_{0 i}
\end{aligned}
$$

where $g_{i}$ is person $i$ 's gender, or a dummy coded 1 for women and 0 for men; $b_{i}$ denotes person $i$ 's year of birth, $d_{i}$ is his or her educational attainment and $r_{i}$ refers to the region where $i$ lives. Finally, $w_{k, i}$ is the individual-specific mean of the $k$-th independent variable. The only individual-level time-invariant variable that we treat as substantively interesting here, however, is gender.

In the model above, the term $u_{0} i$ should be seen in terms of the betweenperson variance that cannot be attributed to the individual-level variables that we added to the model. As shown in Table 1, the estimate of the variance of $u_{0 i}$ in the null model (Model 0 in Table 1) is 0.145 , which is the same as the residual variance. We can conclude, therefore, that differences between individuals account for $0.145 /(0.145+0.145)=0.5$ of the total variance in the dependent variable. At the same time, the estimate of the variance of $u_{0 i}$ in the extended model (Model 1 in Table 2) is 0.033 , whereas the residual variance for that model is 0.143 . This means that the "unexplained" part of the total variance that is due to differences between individuals is $0.033 /(0.033+0.143)=$ 0.188 in the extended model. Adding the individual-level variables to the model considerably reduces the amount of between-person variance. The individuallevel variables in Model 1 account for $1-0.033 / 0.145=0.772$ of the betweenperson variance.

We extend the model further by adding time-varying characteristics, such as age (and its square), tenure (and its square), overall job experience (and its square), weekly working hours, occupational prestige, as well as dummies for living in a rural area, for living in a big city, and for being married, and the number of children (which serves as our measure of the parenthood status). Note that since the model includes both the time-varying characteristics and their individual-specific means, the coefficients for the former are interpreted as within-person effects (Staff and Mortimer 2012). The point estimates of these coefficients are identical to those in a fixed effect model with the same timevarying predictors (a comparison of the two models is available as Table 6 in 
the Appendix):

$$
\begin{aligned}
& y_{i t}=\beta_{0 i}+\beta_{1 i} \text { age }_{i t}+\beta_{2 i} \text { age }_{i t}^{2}+\beta_{3 i} \text { tenure }_{i t}+\beta_{4 i} \text { tenure }_{i t}^{2}+\beta_{5 i} \text { exp }_{i t}+ \\
& \beta_{6 i} \text { exp }_{i t}^{2}+\beta_{7 i} \text { hours }_{i t}+\beta_{8 i} \text { prestige }_{i t}+\beta_{9 i} \text { rural }_{i t}+ \\
& \beta_{10 i} \text { city }_{i t}+\beta_{11 i} \text { married }_{i t}+\beta_{12 i} \text { children }_{i t}+\epsilon_{i t} \\
& \beta_{0 i}=\gamma_{00}+\gamma_{01} g_{i}+\gamma_{02} b_{i}+\gamma_{03} d_{i}+\gamma_{04} r_{i}+\sum_{k=5}^{K} \gamma_{0 k} w_{k, i}+u_{0 i} \\
& \beta_{k i}=\gamma_{k}, \quad k=1, \ldots, 11 \\
& \beta_{12 i}=\gamma_{120}+\gamma_{121} g_{i}+u_{12}
\end{aligned}
$$

In this model formulation, the only coefficient that varies across respondents is that representing the number of children, $\beta_{12 i}$; all other coefficients are set to a fixed value for all respondents. The model thus postulates that the coefficient for children, $\beta_{12 i}$, varies across individuals and at least some part of the variability in the slope is accounted for by gender. The model is based on the assumption that the coefficient for the number of children is different for men than for women. It has two terms involving the number of children: its main effect and its interaction with gender. The latter is a cross-level interaction term, as gender and the number of children are observed at different levels. If the main effect is positive, it means that men benefit from having children in the sense that their earnings go up with each additional child they have. If the interaction effect differs from 0 , it means that children affect women's earnings differently than they do men. Specifically, if the interaction coefficient is negative, it means that women benefit less from having children than do men. If, additionally, its absolute value exceeds that of the main effect, it means that women are penalized for having children.

\subsection{Results}

Table 2 presents the results of our analysis. Model 0 is the empty model, Model 1 accounts only for the individual-level variables and the individual-specific means of the time-varying variables. Model 2 is the full model. In the table, we only present a subset of the full model parameters. They include coefficients for gender and for the time-varying characteristics, which are interpreted as withinperson effects. The table with all coefficients is available in the Appendix (see Table 5).

Working longer hours and working in more prestigious occupations both have a positive effect on earnings. A five hours increase in the number of weekly hours spent at work is associated with an increase in earnings of $2.8 \%$. Ten points on the occupational prestige scale translates into an increase in earnings of $4.8 \%$. One additional year of job experience leads to an increase in earnings of $2.8 \%$, although that effect tends to get weaker over time, as evidenced by the statistically significant coefficient of the square of experience. Tenure, however, 
Table 2: Estimates of the parameters in the two-level hierarchical models fit to POLPAN data

\begin{tabular}{|c|c|c|c|}
\hline & Model 0 & Model 1 & Model 2 \\
\hline Gender & & $\begin{array}{c}-0.304^{* * *} \\
(0.017)\end{array}$ & $\begin{array}{c}-0.282^{\text {*** }} \\
(0.020)\end{array}$ \\
\hline Age & & & $\begin{array}{c}0.022^{*} \\
(0.009)\end{array}$ \\
\hline Age squared & & & $\begin{array}{r}-0.000 \\
(0.000)\end{array}$ \\
\hline Tenure & & & $\begin{array}{c}0.002 \\
(0.002)\end{array}$ \\
\hline Tenure squared & & & $\begin{array}{r}-0.000 \\
(0.000)\end{array}$ \\
\hline Job experience & & & $\begin{array}{l}0.028^{* * *} \\
(0.006)\end{array}$ \\
\hline Job exp. squared & & & $\begin{array}{r}-0.000^{*} \\
(0.000)\end{array}$ \\
\hline Weekly working hours & & & $\begin{array}{l}0.006^{* * *} \\
(0.001)\end{array}$ \\
\hline Job prestige & & & $\begin{array}{l}0.005^{* * *} \\
(0.001)\end{array}$ \\
\hline Rural dummy & & & $\begin{array}{c}0.101^{*} \\
(0.044)\end{array}$ \\
\hline Large city dummy & & & $\begin{array}{c}0.058 \\
(0.059)\end{array}$ \\
\hline Married & & & $\begin{array}{c}0.028 \\
(0.023)\end{array}$ \\
\hline Children & & & $\begin{array}{c}0.014 \\
(0.011)\end{array}$ \\
\hline Gender $\times$ Children & & & $\begin{array}{r}-0.029^{*} \\
(0.013) \\
\end{array}$ \\
\hline AIC & 5662.433 & 4676.583 & 3693.435 \\
\hline $\mathrm{BIC}$ & 5681.432 & 4841.242 & 3953.089 \\
\hline Log Likelihood & -2828.216 & -2312.291 & -1805.718 \\
\hline Num. obs. & 4159 & 4159 & 4159 \\
\hline Num. groups: polpanid & 1422 & 1422 & 1422 \\
\hline Var: polpanid (Intercept) & 0.145 & 0.033 & 0.053 \\
\hline Var: Residual & 0.145 & 0.143 & 0.092 \\
\hline Var: polpanid ntotal & & & 0.005 \\
\hline Cov: polpanid (Intercept) ntotal & & & -0.005 \\
\hline
\end{tabular}

${ }^{* * *} p<0.001,{ }^{* *} p<0.01,{ }^{*} p<0.05$ 
Table 3: Gender pay gap by the number of children as predicted by Model 2 in Table 2

\begin{tabular}{cc}
\hline The number of children & Estimated pay gap \\
\hline 0 & 0.246 \\
1 & 0.267 \\
2 & 0.288 \\
3 & 0.308 \\
4 & 0.328 \\
\hline
\end{tabular}

doesn't seem to have much effect on earnings. All in all, occupational prestige, weekly working hours, and on-the-job experience all a have positive, if moderate, impact on earnings.

According to Model 1, women earn $26.2 \%$ less than otherwise similar men. In Model 2, the estimate of the main effect of gender is slightly lower, implying a gender pay gap of $24.6 \%$, but it applies only to respondents without children. For respondents with children, the size of the pay gap increases with each additional child, as shown in Table 3.

More specifically, the size of the motherhood gender pay gap is estimated as $\exp (-0.282-m \cdot 0.029)$, where $m$ is the number of children. Relative to otherwise similar fathers, mothers suffer an extra penalty of roughly $3 \%$ of their earnings per child, in addition to the gender penalty.

Interestingly, while parenthood further disadvantages women relative to men, we do not find sufficient evidence of fatherhood premia or motherhood penalties. The main effect of the number of children is positive, while its interaction with gender is negative. The latter effect is greater in magnitude than the former, suggesting that men accrue small bonuses for being fathers (i.e., that fathers earn slightly more than otherwise similar childless men), whereas women suffer small penalties for being mothers (i.e., that mothers earn slightly less than otherwise similar women who have no children). However, these effects are too weak to reach statistical significance.

\section{Just gender and motherhood pay gaps in Poland}

In order to estimate just pay gaps, we used a multifactorial (vignette) experimental design. An important advantage of using vignettes in justice and legitimacy research is that they allow for obtaining judgments from respondents concerning combinations of characteristics that occur rather rarely in the population (Auspurg and Hinz 2015). They also allow for nuanced analysis regarding the effect of each characteristic on just earnings evaluations while avoiding most of the cognitive biases associated with traditional survey measures. 


\subsection{Vignettes Sample}

Each participant in our survey was assigned a sample of vignettes. The samples were drawn at random from a population of vignettes. Each vignette described a fictitious full-time employee of certain gender, age, parenthood status, job experience, and occupation. Respondents were asked to evaluate the descriptions in terms of the "appropriateness" of the earnings assigned to the vignettes. Crossing levels of four characteristics — gender, age, occupation, and parenthood status (or the number of children) - creates the population of vignettes. Age of vignette profiles varied between 30 and 65, in 5-year increments. Occupations were classified into three categories: high, medium, and low prestige (using the same PSCO scales as used in the POLPAN study). The prestige scale assigns a score between 0 and 100 to each occupation listed in the classification, with higher scores indicating higher prestige. We grouped the occupations into three broad classes, so that the high category included occupations with a score of 70 or higher, the low category consisted of occupations with a score below 40 , and the middle category included occupations with scores ranging from 40 to 69 . For each level of prestige, we selected seven occupations to include in the vignettes (the list of occupations used and associated prestige scores is listed in the Appendix; see Table 7). Because we wanted vignette samples assigned to respondents to cover the full spectrum of occupational prestige, we used stratified sampling to select a sample for each respondent, with each occupation being a separate stratum. For the number of children, there were three levels: no children; one child; and two or more children.

This means that with 2 gender categories, 8 age levels, 21 occupational titles, and 3 levels of the parenthood status, we had a total of 1,008 vignette combinations. Removing combinations that are logically improbable reduced the total number of available vignettes to 996 .

Apart from the four characteristics listed above, individual vignettes also included information about job experience. That information was added to the vignettes after the vignette population was created and correlated with age. That is, for each vignette, job experience was calculated by adding a small amount of random variation to a linear transformation of age, the transformation being age less years of education. Additionally, for the vignettes in the "two or more children" category, we distinguished between individuals with 2,3 , or 4 children. Vignettes in this category were assigned 2 children with probability $0.50,3$ children with probability 0.35 , and 4 children with probability 0.15 . That way, our vignettes looked more "realistic," since describing individuals as having "two or more children" might have appeared somewhat artificial to our respondents. For vignettes in the "no children" category, no information about the number of children was provided. That is, vignettes in this category were not presented as childless. Instead, no information about children was included. This might have some effect on how our respondents perceived these vignettes, because they could have made implicit assumptions about parenthood status based on other characteristics, such as age and gender, as discussed in conclusions. 
We drew samples of 21 vignettes for each respondent. Because there were 996 unique vignettes and 2,001 participants in our study, each vignette was evaluated by more than one respondent. To each vignette in a vignette sample, we randomly assigned a set of six earnings amounts. These amounts were presented as referring to net monthly on-the-job earnings, defined in the same manner as those in POLPAN, and drawn from a hypothetical distribution, the parameters of which closely resembled those of the actual distribution of earnings in Poland in 2016. Amounts were sampled separately for each vignette and they were listed in a random order. For each amount assigned to a vignette, we asked the respondent how "appropriate" he or she thought this amount would be for the person described in the vignette. Responses were coded on a scale ranging from -5 ("much too low") to 5 ("much too high") with 0 labeled as "appropriate." With 21 vignettes to evaluate, and 6 amounts of earnings assigned to each vignette, each respondent generated 126 evaluations of the justice of earnings.

\subsection{Respondent sample}

The vignette survey was administered to a sample of registered members of an Internet panel run by one of the leading marketing and opinion research companies in Poland in 2017 ( $n=2,001$ respondents). The sample was not random, but it was designed so as to match the composition of the Polish working population in terms of age, gender, education, and geographical location. Approximately half of our respondents were female, with a mean age of 41.4 years.

With 2,001 study participants, and 126 observations generated by each respondent, the total number of justice evaluations in the resulting dataset is 252,126 . However, the size of the dataset that we used in the analyses is much smaller. First, preliminary inspection of the data revealed that some respondents gave "illogical" responses when viewing some of the vignettes. We classified a set of responses as illogical if: $(a)$ the respondent gave identical evaluations to all amounts assigned to the vignette; or $(b)$ there were contradictions in how the respondent evaluated amounts assigned to the vignette. Second, following the procedure proposed by Jasso and Webster (1999), we used the six evaluations of justice for each vignette-respondent combination to estimate a just amount of earnings for that vignette.

After removing the illogical cases and estimating the just amounts, the resulting dataset consists of 38,183 observations from 1,964 respondents' judgments of fair earnings of 996 vignettes.

\subsection{Analytical Strategy}

On the one hand, respondents are likely to differ from one another in their conceptions of what is fair. On the other hand, all respondents are a part of the same culture and are exposed to the same set of cultural beliefs concerning relationships between salient status characteristics and reward levels. Thus, we expect within-respondent variance of the dependent variable to be smaller 
than between-respondent variance, and we expect within-vignette variance of the dependent variable to be smaller than between-vignette variance. The two sources of variability - one associated with the respondent and the other with vignettes - are crossed. Insofar as the same respondent reviewed multiple vignettes and multiple respondents reviewed the same vignette, we use mixedeffects linear models with crossed random effects of respondents and vignettes.

The dependent variable in this study, $y_{i j}$, is the log of net monthly earnings that respondent $i$ thinks is fair for person (vignette) $j$. We use the log transformation, as the distribution of just earnings is highly positively skewed. Similarly as in the POLPAN data analysis, we begin with an "empty" model. The empty model can formally be written as:

$$
y_{i j}=\gamma_{00}+u_{i}+v_{j}+\epsilon_{i j}
$$

Here, $y_{i j}$ is the $\log$ of just earning for person $j$ in the eyes of respondent $i, \gamma_{00}$ is the grand mean of that variable, taken over all respondents and all vignettes, while $u_{i}$ and $v_{j}$ are random effects of respondents and vignettes, respectively. The random effects are assumed to be stochastically independent, so that their covariance is zero, and normally distributed.

According to the estimates in Table 4 (see column labeled Model 0), the proportion of the total variance of the dependent variable that is associated with differences between respondents is equal to $0.095 /(0.095+0.035+0.002)=0.721$, whereas the proportion associated with differences between vignettes is equal to $0.035 /(0.095+0.035+0.002)=0.264$. Differences between respondents account for a greater part of the overall variance than do differences across vignettes. But the share of the overall variance attributed to vignettes is still substantial, meaning that within-vignette differences in just earnings are smaller than between-vignette differences. Adding variables representing vignette characteristics to the model is expected to reduce the between-vignette part of the overall variance.

Just gender pay gaps refer here to differences in the just amounts of earnings between otherwise similar male and female vignettes. To establish the magnitude of these gaps, we first estimate the following model:

$$
\begin{array}{r}
y_{i j}=\gamma_{00}+\gamma_{01} g_{i j}+\gamma_{02} \exp _{i j}+\gamma_{03} \exp _{i j}^{2}+\gamma_{04} \text { prestige }_{i j}+\gamma_{05} \text { children }_{i j}+ \\
\gamma_{06} \text { children }_{i j} g_{i j}+u_{i}+v_{j}+\epsilon_{i j}
\end{array}
$$

In the model, $g_{i j}$ denotes gender in vignette $j$ shown to respondent $i$; gender is a dummy variable coded 1 for women and 0 for men. Similarly, $\exp _{i j}$ refers to job experience (in years) of the person described in vignette $j$ shown to respondent $i$. As we can see, the model above includes terms for job experience and its square, but doesn't include any terms for age. This is because age and job experience in the vignettes correlate at the level of $r=0.99$; including both variables in the model would be redundant. As mentioned above, we use the Polish scale of occupational prestige associated with PSCO to represent the 
Table 4: Estimates of the parameters in the mixed-effects models fit to the vignette data

\begin{tabular}{|c|c|c|}
\hline & Model 0 & Model 1 \\
\hline Constant & $\begin{array}{l}8.473^{* * *} \\
(0.009)\end{array}$ & $\begin{array}{l}8.075^{* * *} \\
(0.017)\end{array}$ \\
\hline Gender & & $\begin{array}{c}-0.014 \\
(0.011)\end{array}$ \\
\hline Job experience & & $\begin{array}{l}0.010^{* * *} \\
(0.001)\end{array}$ \\
\hline Job exp. squared & & $\begin{array}{c}-0.000^{* * *} \\
(0.000)\end{array}$ \\
\hline Job prestige & & $\begin{array}{l}0.005^{\text {*** }} \\
(0.000)\end{array}$ \\
\hline Children & & $\begin{array}{c}0.013^{* *} \\
(0.004)\end{array}$ \\
\hline Gender $\times$ Children & & $\begin{array}{c}-0.001 \\
(0.006)\end{array}$ \\
\hline $\mathrm{AIC}$ & -109708.905 & -110557.208 \\
\hline $\mathrm{BIC}$ & -109674.704 & -110471.706 \\
\hline Log Likelihood & 54858.452 & 55288.604 \\
\hline Num. obs. & 38183 & 38183 \\
\hline Num. groups: respondent ID & 1964 & 1964 \\
\hline Num. groups: vignette ID & 996 & 996 \\
\hline Var: respondent ID (Intercept) & 0.095 & 0.095 \\
\hline Var: vignette ID (Intercept) & 0.035 & 0.014 \\
\hline Var: Residual & 0.002 & 0.002 \\
\hline
\end{tabular}

${ }^{* * *} p<0.001,{ }^{* *} p<0.01,{ }^{*} p<0.05$ 
hierarchy of prestige of vignette occupations. Parenthood status is measured in terms of the number of children, which varies between 0 and 4 . The model also includes an interaction effect between gender and the number of children.

The estimates of variance of the random effects are presented for Model 1 in Table 4. The variance of the random effects associated with respondents is the same as in the baseline "empty" model, as characteristics of the respondents have not yet been introduced into the model. The variance of the random effects of vignettes is lower, however, than in the baseline model: including the vignette characteristics in the extended model reduces the between-vignette variance by $1-0.117 / 0.168=0.304$. That is, the vignette characteristics included in the model account for $30 \%$ of the variance associated with vignettes.

\subsection{Results}

Similarly, as was the case with POLPAN data, job experience and job prestige have positive effects on just earnings: vignette presenting profiles of more prestigious occupations and more on-the-job experience are deemed as deserving higher earnings, all else being equal. Also, the effect of experience declines over time, as evidenced by a negative coefficient for the square of job experience. Yet, these effects are rather weak in absolute terms. The coefficient for gender in Model 1 is negative, but not statistically significant: women are seen as less deserving of earnings than otherwise identical men, but the difference is not large. It is also fairly small in practical terms, equivalent to a pay gap of roughly $1 \%$. This result implies that respondents in the study do not legitimize gender pay gaps.

The main effect of the number of children is positive and statistically significant. However, the interaction term of gender and number of children, while negative, is not significant. Thus, we don't have sufficient evidence to conclude that our respondents believe that the effect of being a parent should affect female employees different than it does male employees.

More specifically, the main effect of the number of children is interpreted in terms of the effect of that variable on the (logged) earnings of male vignette profiles. Of two male profiles, one of which has 1 child and the other no children, the former is seen as deserving $\exp (0.013)=1.013$ times higher earnings than the latter. That is, an additional child translates into a premium of $1 \%$. Given the very small interaction effect between gender and the number of children, the result is essentially identical for female profiles. Respondents judged parents as deserving higher earnings than non-parents, regardless of their gender.

\section{Concluding discussion}

Research on motherhood-related gender pay gaps and motherhood penalties in Poland is sparse. We used a two-step approach to estimate their size and explore whether a sense of propriety - i.e., individuals' acceptance that being a woman and/or a female parent constitutes an appropriate basis for differences 
in pay - explains their persistence. Our results suggest that: $(a)$ although the gender pay gap in Poland is substantial - about 25\% after controlling for job and human capital characteristics; $(b)$ and women in Poland do incur further wage losses when they become mothers, compared to otherwise identical men; (c) mothers do not earn significantly less than women with no children who are similar in terms of experience, occupation, and other relevant job and human capital characteristics. And, finally, neither gender nor motherhood penalties seem to be legitimized.

The results based on POLPAN suggest larger gender pay gaps than the unadjusted gross pay gaps usually reported for Poland (e.g., Eurostat 2017). The gender pay gaps we report, which control for job experience, occupational prestige, and working hours, are also higher than those reported by studies that use cross-sectional data from the Polish Household Survey (e.g. CukrowskaTorzewska and Lovasz 2016).

Research shows that controlling for more productivity and job related characteristics generates larger estimated gender pay gaps in Poland (Goraus-Tańska and Tyrowicz 2014; Majchrowska et al. 2014; Velde, Tyrowicz, and Goraus 2013). In that sense, our estimations are in line with Eurostat's (2017) adjusted and unadjusted gender pay gaps in Poland and other Central and Eastern European countries (Eurostat's unadjusted gaps are smaller than their adjusted gaps, which control only for education). Our estimates control not only for education, but also for a range of other productivity and job-related characteristics. As a result, we estimate larger gaps than does Eurostat and similar analyses. (Note also that our analyses apply only to full time employees, and our data are longitudinal rather than cross-sectional; both may contribute to differences between our estimates and others'.)

On the other hand, our estimates do not match those of Eurostat and others cited above. This might be due to the fact that $(a)$ we include productivity and job related characteristics not available in other datasets; $(b)$ we only analyze gaps for a very specific - full-time employed - sub-sample (to match it with our vignette study); $(c)$ we report the over-time rather than one-point in time gender pay differences.

The Eurostat estimates of adjusted pay gaps show an interesting pattern, which seem to fit our results - the highest gaps are observed in countries "of transition," i.e., those that were part of the Soviet bloc or ex-Soviet republics. Despite current economic differences, these countries share a history of centrally planned economies, in which female labor participation was high and overall earnings inequality low. Still, even during Communism, women were rarely employed in the highest paying jobs (e.g., in the ideologically favored heavy industries) and were mostly employed in service and clerical jobs, as well as so-called light industries (e.g., manufacturing). Women in these countries were, on average, better educated than men, and their representation in certain typically male-dominated occupations (e.g., legal and accounting professions) was high. Additionally, there was a well-developed, state-run network of childcare facilities. The division of household labor was very traditional, however (Mikucka 2013). 
The period of economic transformation solidified gender segregation and seriously weakened states' commitments to maintaining childcare infrastructure. During and after the period of economic "shock-therapy," characterized by high unemployment among both men and women, women were less likely to regain employment (Mikucka 2013). The new cohorts of educated women entering the labor market had to compete for jobs not only with men, but also with older female cohorts. Accepting lower pay, part-time jobs, or job below qualifications under such conditions was common.

Such dynamics may also partly explain why we find little evidence of the motherhood wage penalty or fatherhood wage premium. Historically, parenthood in Poland and other Soviet-bloc countries was not as relevant for labor market outcomes as it was in western nations. This, however, might be changing due to, among other factors, extended paid maternity leaves and abandoned state commitment to childcare infrastructure. Among younger cohorts, motherhood effects might be more pronounced.

Nonetheless, it is important to note that the duration of paid leave and other protections for pregnant women and women with small children (0-3 years old) are more likely to affect short-term, rather than long-term, outcomes (Kleven et al. 2019). Moreover, research shows that gender wage gaps occur before transition to parenthood, calling into question arguments about gender differences in human capital investments (Combet and Oesch 2019).

The results of our vignette study show negative, although not statistically significant, effect of gender (and the interaction of gender with number of children), and a positive effect of children. Such observations indicate the lack of legitimacy for gender inequalities and the legitimacy of the "according to need" justice principle among our respondents. Additionally, they suggest that the relationship between "what is" and "what ought to be" is not as straightforward as suggested by the justification principle.

There are important limitations to our vignette study, however. First, our design might not have been sensitive enough to detect gender and parenthood biases vis-à-vis the perception that earnings in Poland are too low in general. The average fair net earnings according to our respondents should be 5,120 PLN and, at the time of the study, the average gross earnings were 4,272 PLN (ca. 3,042 PLN net). It is thus possible that what our study captured was a general discontent with low earnings. In this case, however, the findings in support of the justification principle (that rely on correlation of two outcome variables generated by similar evaluation process) are especially questionable. That is, although our results do indicate that people find earnings differentials based on such meritocratic characteristics as job experience or occupational status just, we find little evidence that observed inequalities are perceived as such.

Second, we did not systematically vary job experience of the vignette profiles. Legally, a woman on a paid maternity leave is employed and has the same occupational status as she had before the leave. As we were interested in the pure effect of motherhood as a status characteristic, the years of experience in the profiles have not implied any additional breaks to employment beyond random ones. Thus, our results suggest that respondents do not legitimize penalties for 
mothers, or for women in general, when the experience of these women doesn't imply additional career interruptions related to child rearing. This, in turn, suggests that "meritocratic" characteristics (or specific status characteristics) carry more weight than ascribed ones in justice evaluations. González, Cortina, and Rodríguez (2019) report a parallel finding — in their audit study, they found no significant discrimination against highly qualified women with no children, and motherhood mattered mostly in regard to women's chances of being given priority consideration in hiring.

Third, we manipulated parenthood status in a rather subtle way. To increase the internal validity of our study, we instructed the respondents that the vignette profiles are based on actual resumes. Hence, following Correll, Benard, and Paik (2007), we have not flagged childless profiles as such, but rather highlighted the number of children in the "parents" profiles. Considering the traditional perception of a woman's role as a mother, and the historically rooted lack of significant difference between a female-employee and a mother-employee, it is possible that motherhood did not become a salient status characteristic in our study.

Despite those limitations, our analyses of POLPAN results, juxtaposed with the multifactorial experiment data, do suggest that motherhood in Poland does not act independently of gender, and that gender- and parenthood-based inequalities are not perceived as just. This, in turn, implies that additional scope conditions are needed when it comes to the application of the justification principle. Such scope conditions might include, for example, that non-meritocratic social attributes, such as gender and parenthood, only play a role in justice evaluations when differences in productivity characteristics (e.g., job experience) allow for gender-/parenthood-specific attributions, or when the actual wages are not considered outrageously low. Finally, our results suggest that the standard method of correlating responses to the "do-earn"/"should-earn" questions might conflate actual legitimacy with the mechanism that produces it.

\section{A Supplemental material}

\section{A.1 Results for POLPAN data: full table}

Table 5 below is a full version of Table 2 in the main text of our paper; it presents estimates of all the parameters included in the two-level models used in our analysis. The models were estimated using package lme 4 in $\mathrm{R}$ (Bates et al. 2015). 
Table 5: Estimates of the parameters in the two-level hierarchical models fit to POLPAN data

\begin{tabular}{|c|c|c|c|}
\hline & Model 0 & Model 1 & Model 2 \\
\hline Constant & $\begin{array}{l}7^{7.411^{* * *}} \\
(0.012)\end{array}$ & $\begin{array}{c}-44.407^{* * *} \\
(2.565)\end{array}$ & $\begin{array}{c}-44.318^{* * *} \\
(2.531)\end{array}$ \\
\hline Gender & & $\begin{array}{c}-0.304^{* * *} \\
(0.017)\end{array}$ & $\begin{array}{c}-0.282^{* * *} \\
(0.020)\end{array}$ \\
\hline Year of birth & & $\begin{array}{l}0.025^{* * *} \\
(0.001)\end{array}$ & $\begin{array}{l}0.025^{* * *} \\
(0.001)\end{array}$ \\
\hline Vocational education & & $\begin{array}{c}0.061 \\
(0.032)\end{array}$ & $\begin{array}{c}0.066^{*} \\
(0.032)\end{array}$ \\
\hline Secondary education & & $\begin{array}{l}0.091^{* *} \\
(0.035)\end{array}$ & $\begin{array}{l}0.099^{* *} \\
(0.035)\end{array}$ \\
\hline Post-secondary education & & $\begin{array}{c}0.086 \\
(0.047)\end{array}$ & $\begin{array}{r}0.097^{*} \\
(0.047)\end{array}$ \\
\hline Tertiary education & & $\begin{array}{c}0.275^{* * *} \\
(0.047)\end{array}$ & $\begin{array}{l}0.282^{\text {*** }} \\
(0.046)\end{array}$ \\
\hline Region: South & & $\begin{array}{c}-0.024 \\
(0.024)\end{array}$ & $\begin{array}{r}-0.028 \\
(0.024)\end{array}$ \\
\hline Region: East & & $\begin{array}{c}-0.118^{* * *} \\
(0.025)\end{array}$ & $\begin{array}{c}-0.122^{* * *} \\
(0.025)\end{array}$ \\
\hline Region: North-West & & $\begin{array}{c}-0.051 \\
(0.027)\end{array}$ & $\begin{array}{r}-0.052^{*} \\
(0.027)\end{array}$ \\
\hline Region: South-West & & $\begin{array}{c}-0.026 \\
(0.031)\end{array}$ & $\begin{array}{c}-0.029 \\
(0.031)\end{array}$ \\
\hline Region: North & & $\begin{array}{r}-0.068^{*} \\
(0.027)\end{array}$ & $\begin{array}{c}-0.070^{* *} \\
(0.027)\end{array}$ \\
\hline Mean: Age & & $\begin{array}{l}0.065^{* * *} \\
(0.012)\end{array}$ & $\begin{array}{c}0.043^{* *} \\
(0.015)\end{array}$ \\
\hline Mean: Age $^{2}$ & & $\begin{array}{c}-0.000^{* * *} \\
(0.000)\end{array}$ & $\begin{array}{c}-0.000 \\
(0.000)\end{array}$ \\
\hline Mean: Tenure & & $\begin{array}{c}-0.003 \\
(0.005)\end{array}$ & $\begin{array}{r}-0.005 \\
(0.005)\end{array}$ \\
\hline Mean: Tenure ${ }^{2}$ & & $\begin{array}{c}0.000 \\
(0.000)\end{array}$ & $\begin{array}{c}0.000 \\
(0.000)\end{array}$ \\
\hline Mean: Job experience & & $\begin{array}{c}-0.001 \\
(0.006)\end{array}$ & $\begin{array}{c}-0.028^{* * *} \\
(0.008)\end{array}$ \\
\hline Mean: Job experience ${ }^{2}$ & & $\begin{array}{c}0.000 \\
(0.000)\end{array}$ & $\begin{array}{c}0.000 \\
(0.000)\end{array}$ \\
\hline Mean: Hours & & $\begin{array}{l}0.007^{* * *} \\
(0.001)\end{array}$ & $\begin{array}{c}0.001 \\
(0.001)\end{array}$ \\
\hline Mean: Prestige & & $\begin{array}{l}0.008^{* * *} \\
(0.001)\end{array}$ & $\begin{array}{l}0.003^{* *} \\
(0.001)\end{array}$ \\
\hline Mean: Rural & & -0.014 & $-0.113^{*}$ \\
\hline
\end{tabular}




\begin{tabular}{|c|c|c|c|}
\hline & Model 0 & Model 1 & Model 2 \\
\hline & & $(0.020)$ & $(0.049)$ \\
\hline \multirow{2}{*}{ Mean: Large city } & & $0.113^{* * *}$ & 0.056 \\
\hline & & $(0.020)$ & $(0.062)$ \\
\hline \multirow[t]{2}{*}{ Mean: Married } & & 0.026 & 0.000 \\
\hline & & $(0.027)$ & $(0.035)$ \\
\hline \multirow[t]{2}{*}{ Mean: Children } & & 0.005 & 0.002 \\
\hline & & $(0.012)$ & $(0.015)$ \\
\hline \multirow[t]{2}{*}{ Age } & & & $0.022^{*}$ \\
\hline & & & $(0.009)$ \\
\hline \multirow[t]{2}{*}{ Age squared } & & & -0.000 \\
\hline & & & $(0.000)$ \\
\hline \multirow[t]{2}{*}{ Tenure } & & & 0.002 \\
\hline & & & $(0.002)$ \\
\hline \multirow[t]{2}{*}{ Tenure squared } & & & -0.000 \\
\hline & & & $(0.000)$ \\
\hline \multirow[t]{2}{*}{ Job experience } & & & $0.028^{* * *}$ \\
\hline & & & $(0.006)$ \\
\hline \multirow[t]{2}{*}{ Job exp. squared } & & & $-0.000^{*}$ \\
\hline & & & $(0.000)$ \\
\hline \multirow[t]{2}{*}{ Weekly working hours } & & & $0.006^{* * *}$ \\
\hline & & & $(0.001)$ \\
\hline \multirow[t]{2}{*}{ Job prestige } & & & $0.005^{* * *}$ \\
\hline & & & $(0.001)$ \\
\hline \multirow[t]{2}{*}{ Rural dummy } & & & $0.101^{*}$ \\
\hline & & & $(0.044)$ \\
\hline \multirow[t]{2}{*}{ Large city dummy } & & & 0.058 \\
\hline & & & $(0.059)$ \\
\hline \multirow[t]{2}{*}{ Married } & & & 0.028 \\
\hline & & & $(0.023)$ \\
\hline \multirow[t]{2}{*}{ Children } & & & 0.014 \\
\hline & & & $(0.011)$ \\
\hline \multirow[t]{2}{*}{ Gender $\times$ Children } & & & $-0.029^{*}$ \\
\hline & & & $(0.013)$ \\
\hline $\mathrm{AIC}$ & 5662.433 & 4676.583 & 3693.435 \\
\hline $\mathrm{BIC}$ & 5681.432 & 4841.242 & 3953.089 \\
\hline Log likelihood & -2828.216 & -2312.291 & -1805.718 \\
\hline No. of observations & 4159 & 4159 & 4159 \\
\hline No. of respondents & 1422 & 1422 & 1422 \\
\hline $\operatorname{Var}\left(u_{0 i}\right)$ & 0.145 & 0.033 & 0.053 \\
\hline $\operatorname{Var}\left(\epsilon_{i t}\right)$ & 0.145 & 0.143 & 0.092 \\
\hline $\operatorname{Var}\left(u_{12 i}\right)$ & & & 0.005 \\
\hline $\operatorname{Cov}\left(u_{0 i}, u_{12 i}\right)$ & & & -0.005 \\
\hline
\end{tabular}




\section{A.2 Model comparison}

Table 6 compares estimates in a two-level hierarchical model with estimates in a corresponding fixed-effects model. The latter contains the same time-varying independent variables as the former; as we can see from the table, the estimates of coefficients for these variables are exactly the same. The two-level model was estimated using package lme4 in R (Bates et al. 2015). The fixed-effects model, in turn, was estimated using package plm (Millo 2017).

Table 6: A comparison of a two-level hierarchical model with a corresponding fixed-effects model

\begin{tabular}{lcc}
\hline & Two-level model & Fixed-effects model \\
\hline Constant & $-44.383^{* * *}$ \\
Gender & $(2.523)$ \\
& $-0.304^{* * *}$ \\
Year of birth & $(0.017)$ \\
& $0.025^{* * *}$ \\
Vocational education & $0.001)$ \\
& 0.060 \\
Secondary education & $(0.031)$ \\
& $0.091^{* *}$ \\
Post-secondary education & $(0.035)$ \\
& 0.089 \\
Tertiary education & $(0.047)$ \\
& $0.276^{* * *}$ \\
Region: South & $(0.046)$ \\
& -0.027 \\
Region: East & $(0.024)$ \\
& $-0.121^{* * *}$ \\
Region: North-West & $(0.025)$ \\
Region: South-West & $-0.053^{*}$ \\
& $(0.027)$ \\
Region: North & -0.030 \\
& $(0.031)$ \\
Mean: Age & $-0.070^{* *}$ \\
Mean: Age & $(0.027)$ \\
Mean: Tenure & $0.042^{* *}$ \\
& $(0.015)$ \\
& -0.000 \\
& $(0.000)$ \\
& -0.006 \\
& $(0.005)$ \\
\hline
\end{tabular}




\begin{tabular}{|c|c|c|}
\hline & Two-level model & Fixed-effects model \\
\hline Mean: Tenure ${ }^{2}$ & $\begin{array}{c}0.000 \\
(0.000)\end{array}$ & \\
\hline Mean: Job experience & $\begin{array}{c}-0.028^{* * *} \\
(0.008)\end{array}$ & \\
\hline Mean: Job experience ${ }^{2}$ & $\begin{array}{c}0.000 \\
(0.000)\end{array}$ & \\
\hline Mean: Hours & $\begin{array}{c}0.001 \\
(0.001)\end{array}$ & \\
\hline Mean: Prestige & $\begin{array}{l}0.003^{* *} \\
(0.001)\end{array}$ & \\
\hline Mean: Rural & $\begin{array}{c}-0.109^{*} \\
(0.048)\end{array}$ & \\
\hline Mean: Large city & $\begin{array}{c}0.066 \\
(0.061)\end{array}$ & \\
\hline Mean: Married & $\begin{array}{c}0.004 \\
(0.035)\end{array}$ & \\
\hline Mean: Children & $\begin{array}{c}0.004 \\
(0.015)\end{array}$ & \\
\hline Age & $\begin{array}{c}0.023^{*} \\
(0.009)\end{array}$ & $\begin{array}{c}0.023^{*} \\
(0.009)\end{array}$ \\
\hline Age squared & $\begin{array}{r}-0.000^{*} \\
(0.000)\end{array}$ & $\begin{array}{r}-0.000^{*} \\
(0.000)\end{array}$ \\
\hline Tenure & $\begin{array}{c}0.003 \\
(0.002)\end{array}$ & $\begin{array}{c}0.003 \\
(0.002)\end{array}$ \\
\hline Tenure squared & $\begin{array}{c}-0.000 \\
(0.000)\end{array}$ & $\begin{array}{c}-0.000 \\
(0.000)\end{array}$ \\
\hline Job experience & $\begin{array}{l}0.029^{* * *} \\
(0.006)\end{array}$ & $\begin{array}{l}0.029^{* * *} \\
(0.006)\end{array}$ \\
\hline Job exp. squared & $\begin{array}{r}-0.000^{*} \\
(0.000)\end{array}$ & $\begin{array}{r}-0.000^{*} \\
(0.000)\end{array}$ \\
\hline Weekly working hours & $\begin{array}{l}0.006^{* * *} \\
(0.001)\end{array}$ & $\begin{array}{l}0.006^{* * *} \\
(0.001)\end{array}$ \\
\hline Job prestige & $\begin{array}{l}0.005^{* * *} \\
(0.001)\end{array}$ & $\begin{array}{l}0.005^{* * *} \\
(0.001)\end{array}$ \\
\hline Rural dummy & $\begin{array}{c}0.096^{*} \\
(0.044)\end{array}$ & $\begin{array}{c}0.096^{*} \\
(0.044)\end{array}$ \\
\hline Large city dummy & $\begin{array}{c}0.046 \\
(0.058)\end{array}$ & $\begin{array}{c}0.046 \\
(0.058)\end{array}$ \\
\hline Married & $\begin{array}{c}0.025 \\
(0.023)\end{array}$ & $\begin{array}{c}0.025 \\
(0.023)\end{array}$ \\
\hline Children & $\begin{array}{c}-0.001 \\
(0.009)\end{array}$ & $\begin{array}{c}-0.001 \\
(0.009)\end{array}$ \\
\hline $\mathrm{AIC}$ & 3694.241 & \\
\hline
\end{tabular}




\begin{tabular}{lcc}
\hline & Two-level model & Fixed-effects model \\
\hline BIC & 3934.897 & \\
Log Likelihood & -1809.121 & \\
Num. obs. & 4159 & 4159 \\
Num. groups: polpanid & 1422 & \\
Var: polpanid (Intercept) & 0.049 & \\
Var: Residual & 0.096 & 0.340 \\
$\mathrm{R}^{2}$ & & -0.007 \\
Adj. $\mathrm{R}^{2}$ & & \\
\hline$* * * p<0.001{ }^{* *} p<0.01{ }^{*} p<0.05$ &
\end{tabular}

\section{A.3 List of occupations and corresponding prestige scores in the vignette study}

The Polish Sociological Classification of Occupations was originally developed in order to provide a suitable scheme for stratification analyses in Central and Eastern European countries, to which schemes and classifications devised for western countries (e.g., ISCO) did not apply. The first version was developed in 1978 and it was updated several times over the years to reflect the changes in the occupational structure that occurred during and after the economic transition.

The most recent version (Domański, Słomczyński, and Sawiński 2009) of PSCO consists of 260 basic occupational categories. Several additional scales were constructed: Skill Requirements, Complexity of Work, Material Remuneration, and Prestige.

Currently, ISCO is perfectly suitable for analyzing contemporary stratification processes in Poland. However, as early waves of POLPAN rely on PSCO, and we wanted occupational prestige categories in our vignette study to match those in POLPAN, we decided to use the PSCO Prestige Scale. Research shows that this scale has better diagnostic value in Polish context than SIOPS (Standard International Occupational Prestige Scale).

We used the Prestige Scale to select 21 occupations categorized into high, medium-, and low prestige occupations. We categorized occupations with prestige of up to 40 point as low, between 41 and 70 as medium, and above 70 points as high. From each group, we selected occupations based on the following objectives:

1. Selected occupations should maximize the differences between the prestige categories (i.e., be of clearly different prestige to the respondents);

2. Selected occupations within a category should be sufficiently different from each other;

3. Selected occupations should allow for classification into female- and maledominated vs. gender-neutral jobs. 
Table 7: Occupations and corresponding prestige scores

\begin{tabular}{lcc}
\hline Occupation & Prestige score & Prestige category \\
\hline Professor of early education & 97.3 & High \\
Anesthesiologist & 87.8 & High \\
Actor in a theatre & 86.9 & High \\
Radio news presenter & 81.2 & High \\
Plane pilot & 80.6 & High \\
IT security specialist & 77.5 & High \\
Artistic director in an ad agency & 72.7 & High \\
Animal technician & 66.1 & Medium \\
Librarian & 61.3 & Medium \\
Train dispatcher & 60.9 & Medium \\
Nurse & 55.9 & Medium \\
Customs officer & 54.9 & Medium \\
Real estate agent & 51.4 & Medium \\
Accountant & 50.9 & Medium \\
Cashier in a supermarket & 27.6 & Low \\
Mailperson & 27.5 & Low \\
Waitperson & 25.7 & Low \\
Hairdresser & 21.2 & Low \\
Telemarketer & 18.0 & Low \\
Janitor & 10.4 & Low \\
Cloakroom attendant & 8.6 & Low \\
\hline
\end{tabular}

\section{References}

Abendroth, Anja-Kristin, Matt L. Huffman, and Judith Treas. 2014. "The parity penalty in life course perspective: Motherhood and occupational status in 13 European countries." American Sociological Review 79 (5): 993-1014.

Aisenbrey, Silke, and Anette Fasang. 2017. "The interplay of work and family trajectories over the life course: Germany and the United States in comparison." American Journal of Sociology 122 (5): 1448-1484.

Auspurg, Katrin, and Thomas Hinz. 2015. Factorial Survey Experiments. Vol. 175. Thousand Oaks, CA: Sage Publications.

Auspurg, Katrin, Thomas Hinz, and Carsten Sauer. 2017. "Why should women get less? Evidence on the gender pay gap from multifactorial survey experiments." American Sociological Review 82 (1): 179-210.

Bates, Douglas, Martin Mächler, Ben Bolker, and Steve Walker. 2015. "Fitting Linear Mixed-Effects Models Using lme4." Journal of Statistical Software 67 (1): 1-48. 
Budig, Michelle J., Joya Misra, and Irene Boeckmann. 2012. "The motherhood penalty in cross-national perspective: The importance of work-family policies and cultural attitudes." Social Politics 19 (2): 163-193.

. 2016. "Work-family policy trade-offs for mothers? Unpacking the crossnational variation in motherhood earnings penalties." Work and Occupations 43 (2): 119-177.

Charles, Maria. 2011. "A world of difference: International trends in women's economic status." Annual Review of Sociology 37:355-371.

Combet, Benita, and Daniel Oesch. 2019. "The gender wage gap opens long before motherhood. Panel evidence on early careers in Switzerland." European Sociological Review 35 (3): 332-345.

Cooke, Lynn Prince. 2014. "Gendered parenthood penalties and premiums across the earnings distribution in Australia, the United Kingdom, and the United States." European Sociological Review 30 (3): 360-372.

Correll, Shelley J, Stephen Benard, and In Paik. 2007. "Getting a job: Is there a motherhood penalty?" American Journal of Sociology 112 (5): 1297-1338.

Cuddy, Amy J. C., Susan T. Fiske, and Peter Glick. 2004. "When professionals become mothers, warmth doesn't cut the ice." Journal of Social Issues 60 (4): 701-718.

Cukrowska-Torzewska, Ewa. 2016. "How much does it cost to rear children in Poland?" European Journal of Women's Studies 23 (2): 200-216.

Cukrowska-Torzewska, Ewa, and Anna Lovasz. 2016. "Are children driving the gender wage gap? Comparative evidence from Poland and Hungary." Economics of Transition 24 (2): 259-297.

Della Fave, L. Richard. 1986. "Toward an explication of the legitimation process." Social Forces 65 (2): 476-500.

Domański, Henryk, Kazimierz M. Słomczyński, and Zbigniew Sawiński. 2009. Sociological Tools Measuring Occupations: New Classification and Scales. Warsaw: IFiS Publishers.

England, Paula, Jonathan Bearak, Michelle J. Budig, and Melissa J. Hodges. 2016. "Do highly paid, highly skilled women experience the largest motherhood penalty?" American Sociological Review 81 (6): 1161-1189.

Eriksson, Kimmo, and Brent Simpson. 2012. "What do Americans know about inequality? It depends on how you ask them." Judgement and Decision Making 7 (6): 741-745.

Eurostat. 2017. "Adjusted gender pay gap." Accessed October 24, 2019. https : / / circabc . europa . eu / sd / a / b919f002-6ebb-4cbd-90c8-c0d128f 55459/DSSB-2017-Dec-\%203\%20Adjusted\%20gender\%20pay\%20gap. pdf. 
Eurostat. 2019. "Employment statistics." https ://ec . europa.eu/eurostat/ statistics - explained/index . php?title=Employment_statistics \# Employment_rates_by_sex.2C_age_and_educational_attainment_ level.

Frank, Robert H. 2016. Success and Luck: Good Fortune and the Myth of Meritocracy. Princeton: Princeton University Press.

Fuegen, Kathleen, Monica Biernat, Elizabeth Haines, and Kay Deaux. 2004. "Mothers and fathers in the workplace: How gender and parental status influence judgments of job-related competence." Journal of Social Issues 60 (4): 737-754.

González, M. José, Clara Cortina, and Jorge Rodríguez. 2019. "The role of gender stereotypes in hiring: A field experiment." European Sociological Review 35 (2): 187-204.

Goraus-Tańska, Karolina, and Joanna Tyrowicz. 2014. Gender Wage Gap in Poland ? Can It Be Explained by Differences in Observable Characteristics? Working Paper 2014-11. University of Warsaw, Faculty of Economic Sciences.

Heiserman, Nicholas, and Brent Simpson. 2017. "Higher inequality increases the gap in the perceived merit of the rich and poor." Social Psychology Quarterly 80 (3): 243-253.

Jasso, Guillermina, and Murray Webster Jr. 1997. "Double standards in just earnings for male and female workers." Social Psychology Quarterly 60 (1): $66-78$.

. 1999. "Assessing the gender gap in just earnings and its underlying mechanisms." Social Psychology Quarterly 62 (4): 367-380.

Jost, John T., Mahzarin R. Banaji, and Brian A. Nosek. 2004. "A decade of system justification theory: accumulated evidence of conscious and unconscious bolstering of the status quo." Political Psychology 25 (6): 881-919.

Karpiński, Zbigniew, and Kinga Wysieńska-Di Carlo. 2017. "Indywidualne historie zawodowe: analiza ,przeżycia” na rynku pracy." Chap. 3 in Rozwarstwienie spoteczne: zasoby, szanse i bariery. Polskie badanie panelowe, $1988-$ 2013, edited by Anna Kiersztyn, Danuta Życzyńska-Ciołek, and Kazimierz M. Słomczyński, 45-74. Warsaw: Wydawnictwo IFiS PAN.

Kelley, Jonathan, and Krzysztof Zagórski. 2004. "Economic change and the legitimation of inequality: The transition from socialism to the free market in Poland and Hungary, 1987-1994." Research in Social Stratification and Mobility 22:321-366.

Kelly, Nathan J, and Peter K Enns. 2010. "Inequality and the dynamics of public opinion: The self-reinforcing link between economic inequality and mass preferences." American Journal of Political Science 54 (4): 855-870. 
Kenworthy, Lane, and Leslie McCall. 2008. "Inequality, public opinion and redistribution." Socio-Economic Review 6 (1): 35-68.

Kiersztyn, Anna. 2014. "Income measurement in POLPAN 1988-2013." Paper presented at Polish Panel Survey 1988-2013 Workshop, March.

. 2016. "Employment instability revisited: Are "trash contracts" affecting long-term career trajectories?" Chap. 4 in Dynamics of Social Structure: Poland's Transformative Years, 1988-2013, edited by Kazimierz M. Słomczyński, Irina Tomescu-Dubrow, Danuta Życzyńska-Ciołek, and Ilona Wysmułek, 91-116. IFiS Publishers.

Kleven, Henrik, Camille Landais, Johanna Posch, Andreas Steinhauer, and Josef Zweimüller. 2019. Child penalties across countries: evidence and explanations. Working Paper 25524. National Bureau of Economic Research, February.

Kołczyńska, Marta, and Joseph J. Merry. 2016. "Preferred levels of income inequality in a period of systemic change: Analysis of data from the Polish Panel Survey, POLPAN 1988-2003." Polish Sociological Review 194 (2): 171-189.

Majchrowska, Aleksandra, Pawel Strawinski, Karolina Konopczak, and Agnieszka Skierska. 2014. Why are women paid less than men? An investigation into gender wage gap in Poland. Working Paper 2014-31. University of Warsaw, Faculty of Economic Sciences.

Markovsky, Barry. 1988. "Anchoring justice." Social Psychology Quarterly 51 (3): 213-224.

Mikucka, Małgorzata. 2013. "The transition to insecurity: Employment dynamics and its sociodemographic differentiation." International Journal of Sociology 42 (4): 71-99.

Millo, Giovanni. 2017. "Robust Standard Error Estimators for Panel Models: A Unifying Approach." Journal of Statistical Software 82 (3): 1-27. doi:10 . 18637/jss.v082.i03.

Osberg, Lars, and Timothy Smeeding. 2006. "Fair' inequality? Attitudes toward pay differentials: The United States in comparative perspective." American Sociological Review 71 (3): 450-473.

Ridgeway, Cecilia L, and Shelley J. Correll. 2004. "Motherhood as a status characteristic." Journal of Social issues 60 (4): 683-700.

Ridgeway, Cecilia L., Elizabeth Heger Boyle, Kathy J. Kuipers, and Dawn T. Robinson. 1998. "How do status beliefs develop? The role of resources and interactional experience." American Sociological Review 63 (3): 331-350.

Słomczyński, Kazimierz M., Irina Tomescu-Dubrow, Danuta Życzyńska-Ciołek, and Ilona Wysmułek, eds. 2016. Dynamics of Social Structure: Poland's Transformative Years, 1988-2013. Warsaw: IFiS Publishers. 
Staff, Jeremy, and Jeylan T. Mortimer. 2012. "Explaining the motherhood wage penalty during the early occupational career." Demography 49 (1): 1-21.

Velde, Lucas van der, Joanna Tyrowicz, and Karolina Goraus. 2013. What is the true gender wage gap? A comparative analysis using data from Poland. Working Paper 2013-28. Warsaw University, Faculty of Economic Sciences.

Walker, Henry A, George M. Thomas, and Morris Zelditch Jr. 1986. "Legitimation, endorsement, and stability." Social Forces 64 (3): 620-643.

Wysieńska-Di Carlo, Kinga, and Zbigniew Karpiński. 2015. "Is The Motherhood Penalty Real? The Evidence From Poland." Accessed October 24, 2019. https : / / www . shankerinstitute . org/blog / motherhood - penalty real-evidence-poland. 